\title{
Exostosis of Rib: Case Report of an Extremely Rare Giant Osteochondroma at an Unusual Site, Radiopathological Correlation with Brief Review of Literature
}

\author{
Sarita Jilowa ${ }^{1}$ Purnima Paliwal ${ }^{2}$ Arun Kapur ${ }^{3}$ \\ ${ }^{1}$ Department of Radio-diagnosis, Atal Bihari Vajpayee Institute of \\ Medical Sciences and Dr. RML Hospital, New Delhi, India \\ 2 Department of Pathology, Atal Bihari Vajpayee Institute of Medical \\ Sciences and Dr. RML Hospital, New Delhi, India \\ ${ }^{3}$ Department of Surgery, Lady Hardinge Medical College and Dr. RML \\ Hospital, New Delhi, India \\ ${ }^{4}$ Department of Orthopedics, Sanjay Gandhi Memorial Hospital, New \\ Delhi, India
}

G. C. Verma ${ }^{4}$

Address for correspondence Sarita Jilowa, Department of Radiodiagnosis, Atal Bihari Vajpayee Institute of Medical Sciences and Dr RML Hospital, New Delhi, India (e-mail: saritajilowa@gmail.com).

Indian J Radiol Imaging 2021;31:740-744.

\begin{abstract}
Osteochondromas are common benign tumors developing as an abnormal bony growth in the metaphyseal region. Being more of a developmental anomaly rather than a true neoplasm, they are usually found around the growth plates of long bones such as the knee, hip, and shoulder. These are typically managed conservatively if they are asymptomatic; however, they require excision in symptomatic patients. A 38-yearold woman presented with a huge swelling causing disfigurement measuring $16 \times 16 \mathrm{~cm}$ on the left side of chest wall. Radiographs and computed tomography scan showed a bony outgrowth at costochondral junction of second rib which was in continuity with the periosteum. Excision via mediastinal sternotomy and left thoracot-

Keywords

- costochondral junction

- giant osteochondroma

- ribs

- solitary exostosis omy was done. Histopathological features corroborated with the radiological diagnosis of osteochondroma. Osteochondroma should be considered in the differential diagnosis of chest wall tumors. Rib is an extremely rare site of presentation. The cartilaginous cap becomes fully ossified and is lost in longstanding lesions. Huge tumors at such a location can cause irritation of adjacent viscera which can lead to pleural effusion or hemothorax; therefore, a cautious and logical approach to diagnosis is warranted for appropriate therapeutic management.
\end{abstract}

\section{Introduction}

Benign tumors commonly encountered in the ribs are fibrous dysplasia and enchondroma. ${ }^{1}$ Osteochondromas arise from the metaphysis and constitute 20 to $50 \%$ of all benign bone tumors and 10 to $15 \%$ of all bone tumors. These commonly develop during the first decade of life but stop growing when the individual reaches skeletal maturity. Males are affected 1.5 times more commonly than females. ${ }^{2}$ The tumor commonly occurs in long bones but rarely affects ribs with an incidence of 2 to $8 \%{ }^{3}$ The rib tumors are usually small, and occurrence of a giant costal osteochondroma is rare in adults. ${ }^{4-6}$

This case is unique for large size, longstanding at an unusual location, yet lack of malignant transformation. We
DOI https://doi.org/ 10.1055/s-0041-1736406 ISSN 0971-3026. (c) 2021. Indian Radiological Association. All rights reserved.

This is an open access article published by Thieme under the terms of the Creative Commons Attribution-NonDerivative-NonCommercial-License, permitting copying and reproduction so long as the original work is given appropriate credit. Contents may not be used for commercial purposes, or adapted, remixed, transformed or built upon. (https://creativecommons.org/ licenses/by-nc-nd/4.0/)

Thieme Medical and Scientific Publishers Pvt. Ltd., A-12, 2nd Floor, Sector 2, Noida-201301 UP, India 
encountered no such gigantic osteochondroma of rib which possessed a diagnostic challenge due to undetectable cartilage cap which was completely ossified.

\section{Case History}

A 38-year-old woman presented with a 15 -year history of swelling on left side of chest wall which had gradually progressed to the present size of $16 \times 16 \mathrm{~cm}$. Initially, she noted swelling of $2 \times 2 \mathrm{~cm}$ in size which progressed to present size measuring $16 \times 16 \mathrm{~cm}$ causing disfigurement. Some area of skin ulceration was noted along with a discharging sinus over the swelling. According to the patient, one biopsy was attempted 2 years back which triggered the spurt in growth of swelling. There was no history of trauma to ribs, tuberculosis, hypertension, or diabetes or any surgery in the past. This swelling was not painful, so she was not taking any medication for pain. There was no history of blood discharge from the wound of ulceration. On palpation, it was bony hard and skin was stretched over it but intact.

Chest radiographs (anteroposterior and lateral views) showed a dense, round, well-defined bony outgrowth overlying almost whole of left chest wall showing large extrathoracic component. Lucency over surface corresponded to ulceration and sinus formation. The breast shadow was seen separately (-Fig. 1A, B).
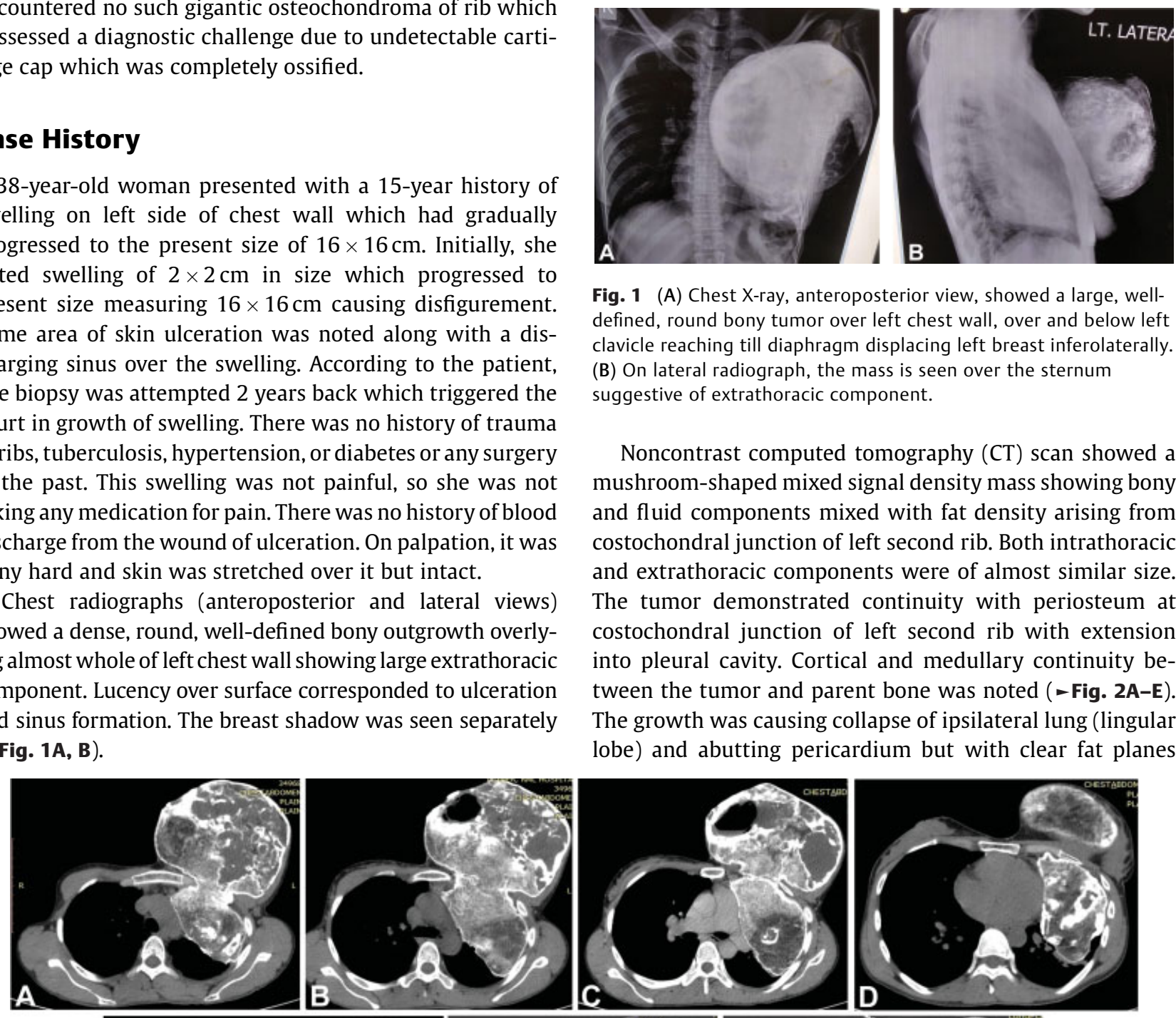

Fig. 1 (A) Chest X-ray, anteroposterior view, showed a large, welldefined, round bony tumor over left chest wall, over and below left clavicle reaching till diaphragm displacing left breast inferolaterally. (B) On lateral radiograph, the mass is seen over the sternum suggestive of extrathoracic component.

Noncontrast computed tomography (CT) scan showed a mushroom-shaped mixed signal density mass showing bony and fluid components mixed with fat density arising from costochondral junction of left second rib. Both intrathoracic and extrathoracic components were of almost similar size. The tumor demonstrated continuity with periosteum at costochondral junction of left second rib with extension into pleural cavity. Cortical and medullary continuity between the tumor and parent bone was noted ( $\mathbf{F i g}$. 2A-E). The growth was causing collapse of ipsilateral lung (lingular lobe) and abutting pericardium but with clear fat planes
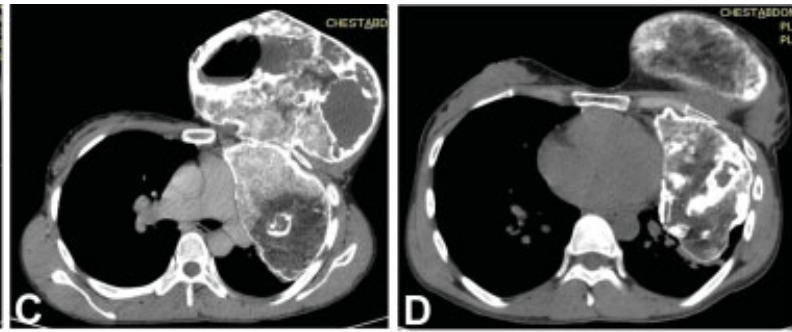
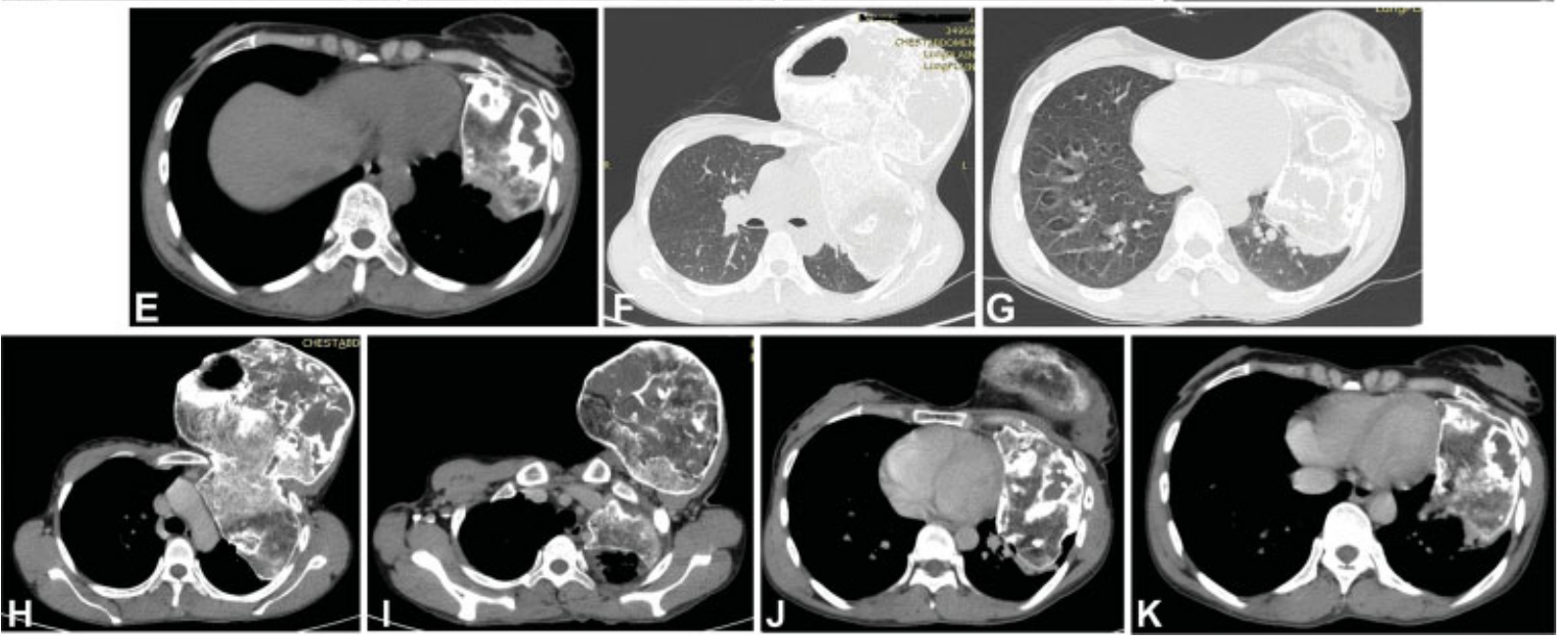

Fig. 2 (A) NCCT of the thorax: Large bony tumor with cystic spaces abutting arch of aorta, origin seen from left second rib. (B) NCCT of the thorax: Dumbbell-shaped tumor with surface sinus. (C) CT of the thorax: Tumor is seen in left hemithorax abutting main pulmonary trunk and left pulmonary artery. (D) NCCT of the thorax: Tumor is abutting pericardium and compressing left lingular lobe bronchus. (E) NCCT of the thorax: Lower extent of the tumor, left breast seen separately. (F) CT of the thorax (lung window): Normal right lung field, consolidation of the left lung adjoining the tumor. (G) CT of the thorax (lung window): Normal right lung field, consolidation of the left lung adjoining the tumor. (H) CECT image shows tumor contents (bony, fluid, and some fat densities) and relationship with arch of aorta. (I) CECT of the thorax: Left subclavian artery in close opposition to medial tumor margin. (J) CECT of the thorax: Close contact of the tumor with pericardium. (K) CECT of the thorax: Lower extent of tumor, clear margin seen with mediastinal structures. CECT, contrast-enhanced computed tomography; CT, computed tomography; NCCT, noncontrast computed tomography. 
(-Fig. 2F, G). Periosteum was intact and there was no evidence of soft tissue infiltration. The mass showed little or no enhancement on contrast CT (-Fig. $\mathbf{2 H - K}$ ).

On magnetic resonance imaging, the mass was of mixed signal intensity on T1- and T2-weighted images with predominantly bony contents with cysts formation. There was a sinus (measuring $6 \times 2.7 \mathrm{~cm}$ ) showing fluid-fluid level. Postcontrast scans revealed minimal or no enhancement in most of tumor parts (-Fig. 3A-G).

Positron emission tomography (PET) scan revealed lowgrade heterogeneous fluorodeoxyglucose (FDG) uptake in the tumor. Low-grade FDG uptake was seen in deeper aspect of calcified mass adjacent to anterior part of left second rib (maximum standard unit value $\left[S V_{\max }\right] 1.3$ ). Increased FDG uptake was also noted in the tissue surrounding the ulceration (SUVmax 2.9). Rest of the masses did not show any significant SUV uptake. Normal metabolic activity was seen in the myocardium. Lungs showed no hypermetabolic pulmonary nodules. There was no active mediastinal, axillary, and hilar lymphadenopathies (-Fig. 4A, B).

All radiologic findings and PET were suggestive of benign primary bony tumor. Diagnosis of osteochondroma was kept after ruling out possibilities of giant cell tumor, atypical fibrous dysplasia, and chondrosarcoma. Considering the large size leading to disfigurement, sinus discharge, compression of left lung causing breathlessness surgery was planned.

Mediastinal sternotomy and "trap-door" thoracotomy were performed with removal of tumor mass into two pieces, along with removal of second and third ribs. Intraoperatively, it was a hard bony dumbbell-shaped mass, $5.5 \mathrm{~kg}$ in weight arising from left second rib and was decorticating third rib. Adjoining left lung was collapsed. Chest wall reconstruction was done with pectoralis major muscle flap.

Postoperative period was uneventful and the postoperative chest X-ray showed air entry into collapsed left lung (-Fig. 5). On 3 years follow-up, patient was doing fine.

Gross specimen examination revealed round hard bony mass of uniform contour with extrathoracic component of $16 \times 16 \mathrm{~cm}$ and intrathoracic component measuring $22 \times 17 \mathrm{~cm}$ (-Fig. 6). Cut section revealed tumor as a mess of bony trabeculae and cavities filled with blood.

Histopathology from intrathoracic component revealed a homogenous picture showing interlacing mature bony trabeculae with intervening hematopoietic to fatty marrow. Sections from hemorrhagic and myxoid areas of extrathoracic tumor showed woven bone, fibrocellular stroma, congested capillary channels, and large areas of hyalinization. Rest of the tumors showed mature bony trabeculae with intervening hematopoietic to fatty marrow. No cartilaginous cap was identified in both components on extensive sampling (-Fig. 7A-D).

Based on clinicoradiopathologic correlation, a diagnosis of osteochondroma was rendered.

\section{Discussion}

Osteochondromas can be solitary or multiple, multiple lesions are usually seen in association with hereditary
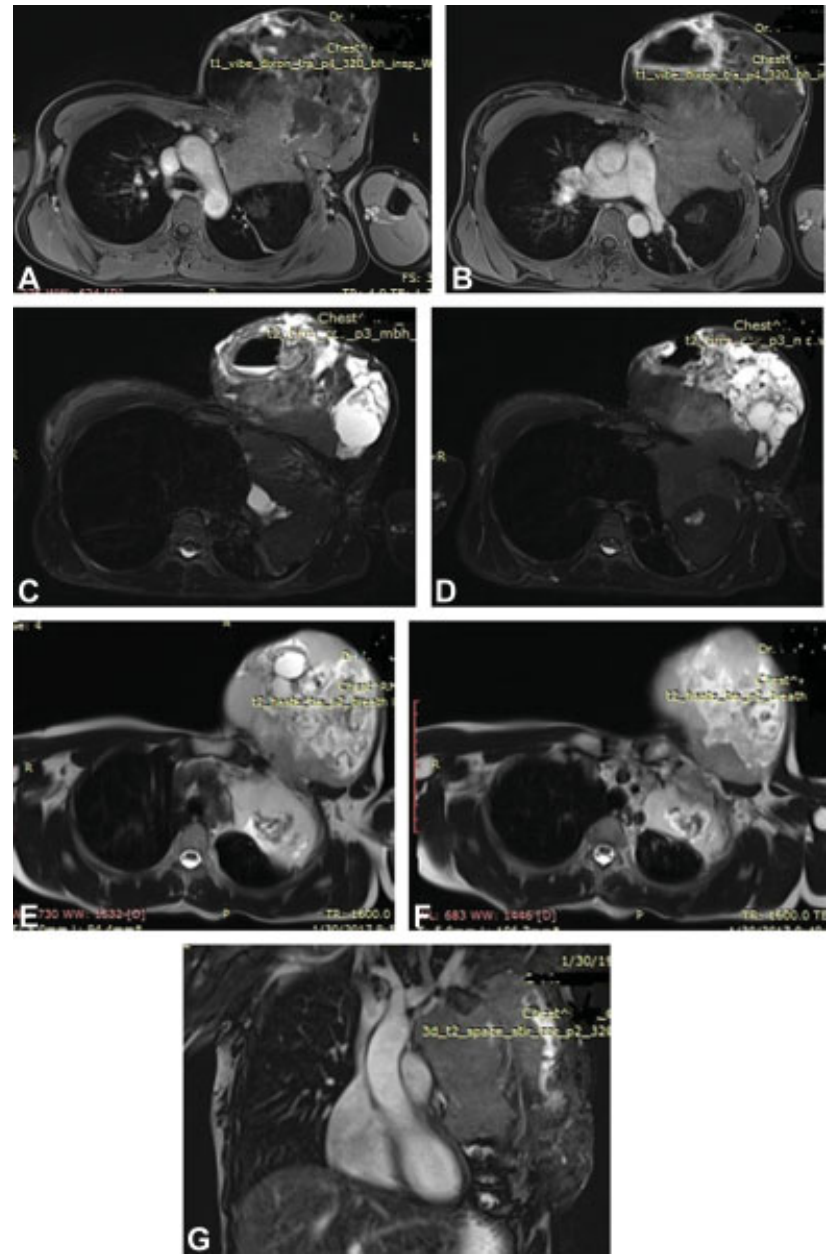

Fig. 3 (A) MRI: 3D T2 space stir tra showing heterogeneous tumor contents at arch level, clear fat planes. (B) MRI: 3D T2 space stir tra, tumor at pulmonary trunk, compressing left pulmonary artery. (C) MRI: T2 tirm tra. Mixed intensity tumor contents showing extrathoracic and intrathoracic extension. (D) MRI: T2 tirm tra. Mixed intensity tumor contents showing extrathoracic and intrathoracic extension. (E) MRI: T2 haste tra. Tumor showing clear fat planes with aortic arch. (F) MRI: T2 haste tra. Tumor closely abutting left subclavian artery, caution for surgeon. (G) MRI: 3D T2 space stir cor. Intrathoracic and extrathoracic tumors showing relations with mediastinal structures and breast. MRI, magnetic resonance imaging.
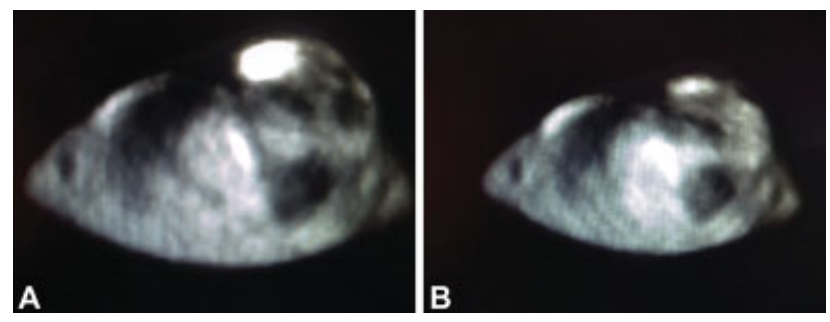

Fig. 4 (A, B) PET image: Increase FDG uptake at sinus site due to active inflammation. No uptake was noted in main tumor suggesting benign etiology. FDG, fluorodeoxyglucose; PET, positron emission tomography.

multiple exostosis (HME). They may present as a pedunculated or sessile mass protruding from the parent bone. The characteristic radiologic findings are of a lesion composed of cortical and medullary bone with an overlying hyaline 


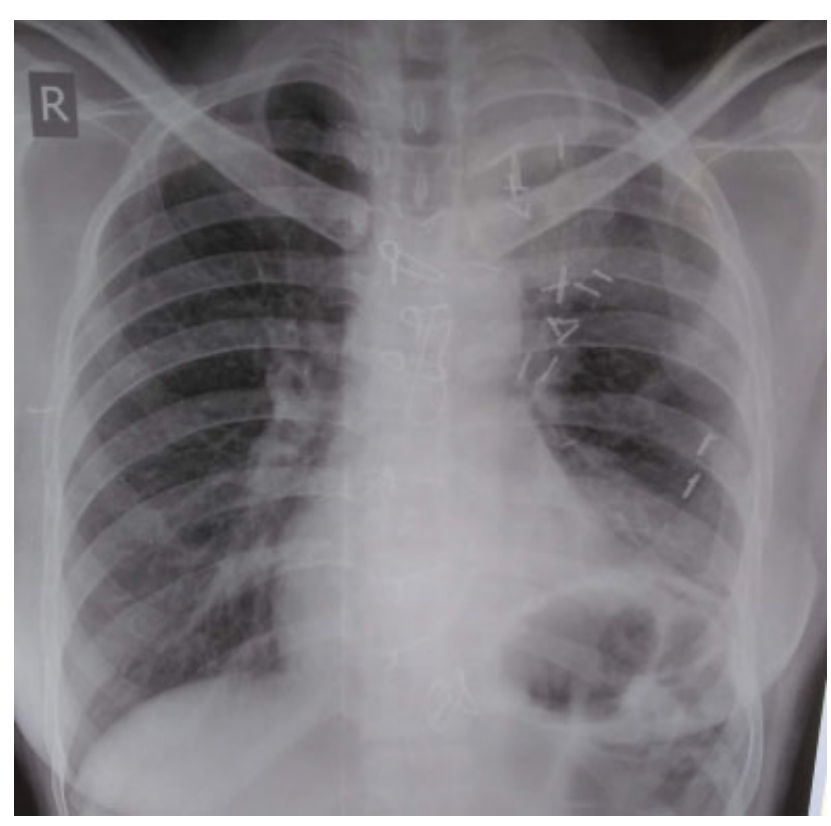

Fig. 5 Postoperative chest X-ray shows good expansion of left lung.

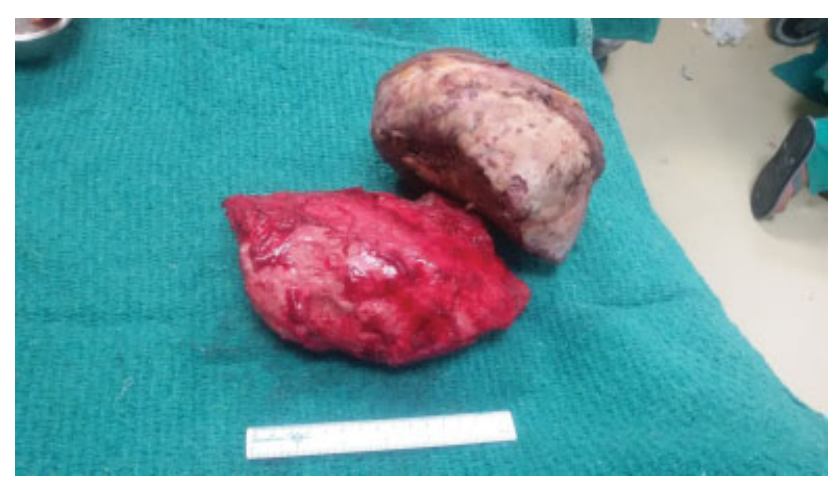

Fig. 6 Gross specimen cut into two pieces (was not possible to remove in whole due to huge size).

cartilage cap. It is a developmental lesion arising when the endochondral bone growth is most active. The ring of Ranvier covering the epiphyseal growth plate is defective, and it is thought that due to cut back remodeling, a fragment of epiphyseal growth plate cartilage protrudes laterally through this defect, instead of its usual descent to metaphysis. Eventually, there is endochondral ossification of this lateral extension which is seen as a cartilage cap covered protrusion on the bony surface. Its cortex is in direct continuity with the cortex of the affected bone, being enveloped by its periosteum. For a diagnosis of osteochondroma to be made, this bony outgrowth must have a direct continuity with the underlying cortex and medullary canal. ${ }^{2}$

The cartilaginous cap has varied morphology, radiologically presenting as either a smooth and uniform contour or as an irregular and bosselated surface with a cauliflower-like appearance. The thickness of this cap changes with increasing age, it may be several centimeters thick in children and adolescents, whereas it may be only a few millimeters in an adult or may be completely absent in some cases. This absence is usually a result of wear and tear abrasion, although it has also been recognized that rarely osteochondromas may lose the cartilaginous cap in very longstanding lesions. The cartilaginous cap in the present case was not discernible even after extensive sectioning. At an advanced age, the cartilaginous cap may become fully ossified so that only an "osseous exostosis" remains without any cartilage., ${ }^{2,7}$

Complete radiological skeletal survey should be performed in cases with multiple osteochondromas to work up for any other significant anomaly and to rule out HME. ${ }^{2}$

In the present case, various differential diagnosis were considered on the basis of histology in the form of hematopoietic pseudotumor, posttraumatic fibro-osseous lesion of ribs, and variant fibrous dysplasia. The diagnosis of hematopoietic pseudotumor was considered in view of abundant hematopoietic marrow and absence of cartilaginous cap. However, this was ruled out based on lack of hematologic abnormalities and unusually large size of lesion. Posttraumatic fibro-osseous lesion of ribs and variant fibrous dysplasia were also ruled out based on lack of classic radiologic findings and presence of abundant hematopoietic marrow, although small foci of woven bone formation, fibrous stroma, and hyalinization were noted.

The diagnosis was strongly supported by the radiologic picture of mushroom-shaped growth arising from anterior region at costochondral junction. Cortical and medullary continuity between osteochondroma and parent bone was characteristic of osteochondroma. ${ }^{2}$

The only other giant costal chondrosarcoma reported by Liu et al was associated with malignant transformation and HME unlike our case. ${ }^{8}$ Alhames and Almhanna have also reported a case of large osteochondroma without any associated family history, but the size is smaller than the present case. ${ }^{9,10}$ The spectrum of complications which may be seen ranges from deformity, fracture, vascular compromise,
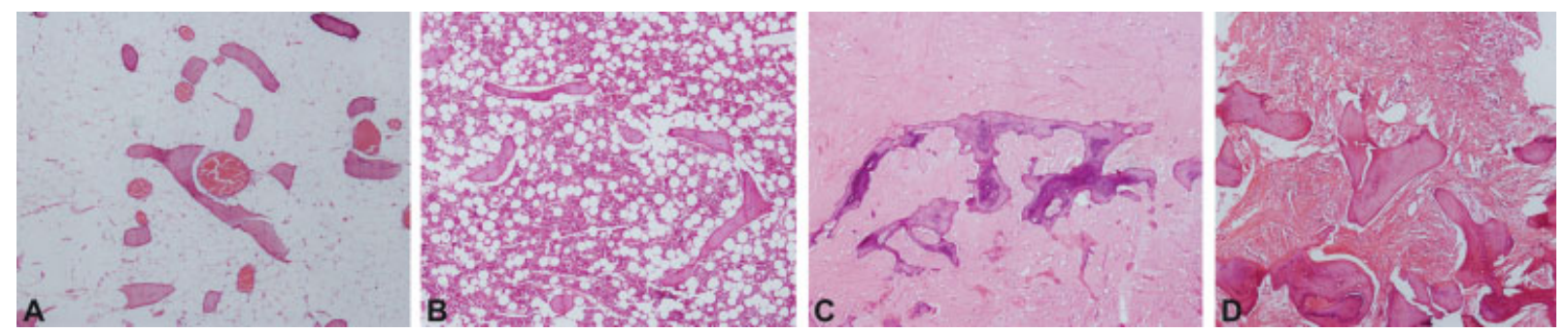

Fig. 7 (A) Histopathology: Bony trabeculae in fatty marrow. (B) HPE: Bony trabeculae in hematopoietic marrow. (C) HPE: Foci of hyalinization and calcification. (D) HPE: Fascicles of spindle cells in marrow spaces. 
neurologic sequelae, overlying bursa formation to malignant transformation in some cases; however, they are more frequent in cases with underlying multiple hereditary exostosis. Malignant transformation is assessed by the presence of a thick cartilage cap more than $1.5 \mathrm{~cm}$ even after attainment of skeletal maturity and a progressive increase in the growth. It is an uncommon occurrence and can be seen in $1 \%$ of solitary osteochondromas and in 3 to $5 \%$ of patients with HME. ${ }^{2}$ There was no evidence of malignant transformation in this case on extensive sampling.

\section{Conclusion}

This case is rare for the large size, unusual location, and yet lack of malignant transformation. Costal osteochondroma is an important condition to recognize due to its complications such as reduced range of movement, pain, cosmetic abnormalities, and bursitis. These may grow into the chest cavity and cause compressive symptoms. The importance of clinicoradiologic correlation and loss of cartilaginous cap in longstanding cases of osteochondroma is exemplified. ${ }^{2,7}$

\section{Declaration of Patient Consent}

The authors certify that they have obtained all appropriate patient consent forms. In the form, the patient(s) has/have given his/her/their consent for his/her/their images and other clinical information to be reported in the journal. The patients understand that their names and initials will not be published and due efforts will be made to conceal their identity, but anonymity cannot be guaranteed.

\section{Financial Support and Sponsorship}

None.

\section{Source of Support}

None.

\section{Conflict of Interest}

None declared.

\section{Acknowledgments}

The authors would like to thank Dr. (Prof.) V.K. Tiwari, burn and plastic surgeon, for guiding surgery in his presence. The authors would also like to thank Dr. Anubhav Gupta, CTVS surgeon, and Dr. Sameek Bhattacharya, plastic surgeon, for accomplishing the task successfully in a team work.

\section{References}

1 Teitebaum SL. Tumors of the chest wall. Surg Gynecol Obstet 1969;129(05):1059-1073

2 Murphey MD, Choi JJ, Kransdorf MJ, Flemming DJ, Gannon FH. Imaging of osteochondroma: variants and complications with radiologic-pathologic correlation. Radiographics 2000;20(05): 1407-1434

3 Waller DA, Newman RJ. Primary bone tumours of the thoracic skeleton: an audit of the Leeds regional bone tumour registry. Thorax 1990;45(11):850-855

4 Kikuchi R, Mino N, Matsukura T, Hirai T. Resected osteochondroma of the rib in an elderly patient. Gen Thorac Cardiovasc Surg 2010;58(11):588-591

5 Tateishi U, Gladish GW, Kusumoto M, et al. Chest wall tumors: radiologic findings and pathologic correlation: part 1. Benign tumors. Radiographics 2003;23(06):1477-1490

6 Lee CY, Ham SY, Oh YW, Lee SH, Kim KT. Osteochondroma arising from a rib mimicking a calcifying anterior mediastinal mass. J Korean RadiolSoc 2007;57:533-535

7 Adler CP. Bone Diseases: Macroscopic, Histological and Radiological Diagnosis of Structural Changes in the Skeleton. Berlin: Springer; 2000

8 Liu W, Kong D, Tang J, Yu F. Giant costal osteochondroma in a man with multiple exostoses. Ann Thorac Surg 2013;96(02):675-677

9 Alhames S, Almhanna K. Large rib osteochondroma in a child in Aleppo, Syria. J Surg Case Rep 2018;2018(09):rjy247

10 Zarqane H, Viala P, Dallaudière B, Vernhet H, Cyteval C, Larbi A. Tumors of the rib. Diagn Interv Imaging 2013;94(11):1095-1108 